\title{
JORDAN SUBALGEBRAS OF BANACH ALGEBRAS
}

\author{
by F. F. BONSALL \\ (Received 15th November 1976)
}

\section{Introduction}

We recall that a $J C$-algebra (Størmer (3)) is a norm closed Jordan algebra of self-adjoint operators on a Hilbert space. Recently, Alfsen, Shultz, and Størmer (1) have introduced a class of abstract normed Jordan algebras called $J B$-algebras, and have proved that every special $J B$-algebra is isometrically isomorphic to a $J C$-algebra. We show that this result brings to a satisfactory conclusion the discussion in (2) of certain wedges $W$ in Banach algebras and their related Jordan algebras $W-W$, and leads to two characterisations of the bicontinuously isomorphic images of $J C$ algebras.

It was proved in (2) that if $W$ is a closed type-0 locally multiplicative wedge in a Banach algebra and the set $\left\{\left\|(1+w)^{-1}\right\|: w \in W\right\}$ is bounded, then $W-W$ is a closed Jordan algebra and behaves in many ways like a $J C$-algebra with positive cone $W$. It can now be seen that $W-W$ is in fact bicontinuously isomorphic to a $J C$-algebra. We prove also that for a closed type-0 wedge $W$ in a Banach algebra, to be locally multiplicative is equivalent to having $x y x \in W$ whenever $x, y \in W$. As a corollary we obtain two characterisations of those Jordan subalgebras $R$ of a Banach algebra that are bicontinuously isomorphic to $J C$-algebras. The first involves subadditivity of the spectral radius $r$, and in the second subadditivity is replaced by a submultiplicative property:

$$
x, y \in R, \lambda \in R \cap \operatorname{Sp}(x y) \Rightarrow|\lambda| \leqslant r(x) r(y) .
$$

For $J C$-algebras $A$ it is obvious that the stronger submultiplicative property

$$
r(x y) \leqslant r(x) r(y) \quad(x, y \in A)
$$

holds, but it remains an open question whether this holds on their bicontinuously isomorphic images $R$.

Finally we show that if $W$ is a closed type- 0 locally multiplicative cone but the set of inverses $(1+w)^{-1}$ is not necessarily bounded, the completion of $W-W$ with respect to the spectral radius norm is a special $J B$-algebra. Thus in this more general case $W-W$ remains isomorphic to a dense Jordan subalgebra of a $J C$-algebra.

\section{Notation}

$B$ will denote a complex Banach algebra with unit, $\operatorname{Inv}(B)$ will denote the set of invertible elements of $B$, and for $a \in B, \operatorname{Sp}(a)$ and $r(a)$ will denote the spectrum and spectral radius of $A$. 
A wedge in $B$ is a non-void subset $W$ of $B$ such that

$$
x, y \in W, \alpha \in \boldsymbol{R}^{+} \Rightarrow x+y, \alpha x \in W .
$$

A wedge $W$ in $B$ is of type-0 if

$$
x \in W \Rightarrow 1+x \in \operatorname{Inv}(B) \text { and }(1+x)^{-1} \in W,
$$

is locally multiplicative if

$$
x, y \in W, x y=y z \Rightarrow x y \in W,
$$

and is a cone if $W \cap(-W)=\{0\}$.

$H$ will denote a complex Hilbert space, and $B L(H)$ the Banach algebra of all bounded linear operators on $H$. A JC-algebra is a real linear subspace $A$ of $B L(H)$, closed with respect to the operator norm, consisting of self-adjoint operators, and satisfying

$$
a, b \in A \Rightarrow a b+b a \in A .
$$

The positive cone $A^{+}$in a $J C$-algebra $A$ is the set of elements of $A$ that are positive operators in the usual sense (that is operators $a$ with $(a x, x) \geqslant o(x \in H)$ ).

Following Alfsen, Shultz and Størmer (1), a $J B$-algebra is a real Banach space $X$ which is a Jordan algebra with respect to a product $x \circ y$, which has a unit element, and which satisfies (for all $x, y \in X$ )

$$
\|x \circ y\| \leqslant\|x\|\|y\|, \quad\left\|x^{2}\right\|=\|x\|^{2}, \quad\left\|x^{2}\right\| \leqslant\left\|x^{2}+y^{2}\right\| .
$$

$X$ is a special $J B$-algebra if it is also a subset of an associative algebra and $x \circ y=\frac{1}{2}(x y+y x)$, where $x y$ is the associative product.

3.

Theorem 1. Let $W$ be a closed type-0 locally multiplicative wedge in $B$, let $R=W-W=\{x-y: x, y \in W\}$, and suppose that the set $\left\{\left\|(1+w)^{-1}\right\|: w \in W\right\}$ is bounded. Then $r$ is a norm on $R$ equivalent to the given norm, and $R$ with the norm $r$ is a special JB-algebra.

Proof. We recall from (2, Theorem 3) that $R$ is a closed real linear subspace and Jordan subalgebra of $B$, that $r$ is subadditive on $R$, that all elements of $R$ have their spectra contained in the reals, and that

$$
W=\left\{x \in R: \operatorname{Sp}(x) \subset R^{+}\right\} .
$$

By (2, Theorem 9), $W \cap(-W)=\{0\}$, and so, by (2, Theorem 4), $r$ is a norm on $R$ satisfying the inequality

$$
r\left(\frac{1}{2}(x y+y x)\right) \leqslant r(x) r(y) \quad(x, y \in R) .
$$

By hypothesis, there exists a real constant $M$ such that

$$
\left\|(1+w)^{-1}\right\| \leqslant M \quad(w \in W) .
$$

We prove that 


$$
\|x\| \leqslant(2\|1\|+3 M) r(x) \quad(x \in R) .
$$

Since $r$ is a norm, this is trivial if $r(x)=0$; and we may therefore suppose, by normalisation, that $r(x)=\frac{1}{2}$. Then $\operatorname{Sp}(1+x) \subset\left[\frac{1}{2}, \frac{3}{2}\right]$. By (1), $1+x$ is therefore of the form $\frac{1}{2}+u$ with $u \in W$. Since $W$ is of type-0 it follows that $(1+x)^{-1} \in W$, and we have $\mathrm{Sp}\left((1+x)^{-1}\right) \subset\left[\frac{2}{3}, 2\right]$. Again by (1), it follows that $(1+x)^{-1}=\frac{2}{3}+w$ with $w \in W$, and so $1+x=\frac{3}{2}\left(1+\frac{3}{2} w\right)^{-1}$. The inequality (2) now gives $\|1+x\| \leqslant \frac{3}{2} M$, and (3) is proved.

We have now proved that $r$ is a norm on $R$ equivalent to the given norm, and so $R$ with the norm $r$ is a real Banach space. It is obvious that $r\left(x^{2}\right)=(r(x))^{2}$ for all $x \in R$, and, since the squares of elements of $R$ belong to $W$, the proof will be complete if we show that

$$
r(u) \leqslant r(u+v) \quad(u, v \in W) .
$$

Let $u, v \in W$. Then $u+v \in W$, and, by (1),

$$
r(u+v)-(u+v) \in W .
$$

Since $W$ is a wedge, it follows that

$$
r(u+v)-u=v+r(u+v)-(u+v) \in W,
$$

and so $\operatorname{Sp}(r(u+v)-u) \subset \boldsymbol{R}^{+}$. Thus (4) is proved, and the proof is complete.

Corollary 2. Let $W, R$ be as in Theorem 1 . Then $R$ is bicontinuously isomorphic to a JC-algebra A, and W corresponds under this isomorphism to the positive cone $\mathrm{A}^{+}$ of $A$.

Proof. By Theorem 1 and (1, Lemmas 9.3 and 9.4), $R$ with the norm $r$ is isometrically isomorphic to a $J C$-algebra $A$. Since the norm $r$ is equivalent on $R$ to the given norm, the isomorphism is bicontinuous with respect to the given norm. The identification of the image of $W$ with $A^{+}$follows at once from the fact that $W$ is the set of squares of elements of $R\left(2\right.$, Theorem 9) and the corresponding fact for $A^{+}$.

Corollary 3. Let $W$ be a closed type-0 locally multiplicative wedge. Then the set $\left\{\left\|(1+w)^{-1}\right\|: w \in W\right\}$ is bounded if and only if $W$ is a normal cone (that is, there exists a constant $\kappa>0$ with $\|x+y\| \geqslant \kappa\|x\|(x, y \in W))$.

Proof. Let $E=\left\{\left\|(1+w)^{-1}\right\|: w \in W\right\}$. By (2, Proposition 10(i)), $E$ is bounded if $W$ is a normal cone. Conversely, suppose that $E$ is bounded. By Theorem 1 there exists a positive constant $\kappa$ with

$$
r(x) \geqslant \kappa\|x\| \quad(x \in R) .
$$

By (2, Proposition 10(ii)), this shows that $W$ is a normal cone.

Theorem 4. Let $W$ be a closed type-0 wedge in $B$. Then the following statements are equivalent:

(i) $W$ is locally multiplicative,

(ii) $x, y \in W \Rightarrow x y x \in W$. 
Proof. That (i) implies (ii) was proved in (2, Theorem 5). Suppose conversely that (ii) holds. Given $w \in W$, it is clear that $w^{n} \in W(n=1,2, \ldots)$, the fact that $1 \in W$ giving the case $n=2$. We prove that

$$
\operatorname{Sp}(w) \subset \boldsymbol{R}^{+}(w \in W) .
$$

The invertibility of $1+w$ gives

$$
\mathrm{Sp}(w) \cap\left(-\boldsymbol{R}^{+}\right) \subset\{0\} \quad(\mathrm{w} \in \mathrm{W}) .
$$

We argue as in the proof of (2, Proposition 1). Suppose that $w \in W$ and that $\rho e^{i \theta} \in \mathrm{Sp}(w)$ with $\rho>0, \theta \in R, 0<|\theta|<\pi$. Choose the greatest positive integer $n$ with $n|\theta|<\pi$. Then $n|\theta| \geqslant \pi / 2$. Take $b=w^{n}$ and observe that $\rho^{n} e^{i n \theta}$ is of the form $-\gamma+i \delta$ with $\gamma \geqslant 0$ and $\delta \in R \backslash\{0\}$. Then $(\gamma+b)^{2} \in W$ and $-\delta^{2} \in \operatorname{Sp}\left((\gamma+b)^{2}\right)$, contradicting (6). Thus $\mathrm{Sp}(w) \subset \boldsymbol{R}$ and (5) follows from (6).

We prove next that

$$
w \in W, r(w)<1 \Rightarrow 1-w \in W .
$$

Given $w \in W$ with $r(w)<1$, we have $(1-w)^{-1}=1+b$ with $b=\sum_{k=1}^{\infty} w^{k} \in W$. Thus $1-w=(1+b)^{-1} \in W$.

Now let $x, y \in W$ with $x y=y x$, and suppose first that $x \in \operatorname{Inv}(B)$ and $r(x)<1$. By (5) we have $r(1-x)<1$, and by (7) $1-x \in W$. Since the binomial series for $(1-t)^{-1 / 2}$ has positive coefficients, we therefore have

$$
x^{-1 / 2}=\{1-(1-x)\}^{-1 / 2} \in W,
$$

and $x^{-1 / 2} y=y x^{-1 / 2}$. By condition (ii), we have in turn $x^{-1 / 2} y x^{-1 / 2} \in W, x^{1 / 2} y x^{1 / 2}=$ $x\left(x^{-1 / 2} y x^{-1 / 2}\right) x \in W$. Therefore

$$
x y=x^{1 / 2} y x^{1 / 2} \in W,
$$

and it is clear that this still holds without the condition $r(x)<1$. Finally, given arbitrary $x, y \in W$ with $x y=y x$ and $\epsilon>0$, we have $\epsilon+x \in W \cap \operatorname{Inv}(B)$, and $(\epsilon+x) y=$ $y(\epsilon+x)$. Therefore $(\epsilon+x) y \in W$. Since $W$ is closed, we have $x y \in W$, and the proof is complete.

Corollary 5. Let $R$ be a Jordan subalgebra of $B$ containing 1 . Then $R$ is bicontinuously isomorphic to a JC-algebra if and only if it satisfies the following conditions:

(i) $R$ is closed,

(ii) $\mathrm{Sp}(x) \subset \boldsymbol{R}(x \in R)$,

(iii) $r$ is subadditive on $R$,

(iv) $\left\{\left\|\left(1+x^{2}\right)^{-1}\right\|: x \in R\right\}$ is bounded.

Proof. Suppose first that $R$ satisfies the stated conditions (i)-(iv), and let $W=\left\{x \in R: S p(x) \subset R^{+}\right\}$. By (2, Theorem 3 ), $W$ is a closed type-0 locally multiplicative wedge and $R=W-W$. Since elements of $W \cap \operatorname{Inv}(B)$ have square roots in $W$, the set $\left\{\left\|(1+w)^{-1}\right\|: w \in W\right\}$ is bounded. By Corollary $2, R$ is bicontinuously isomorphic to a $J C$-algebra.

Conversely, suppose that $\phi$ is a bicontinuous isomorphism of $R$ onto a $J C$-algebra 
$A$, and let $W=\phi^{-1}\left(A^{+}\right)$. Then $W$ is a closed cone and $R=W-W$. If $a, b \in A$ with $a b=b a=1$, and $x=\phi^{-1}(a), y=\phi^{-1}(b)$, then $x y=y x=1$. This is not quite obvious since $\phi$ is not an isomorphism for the associative structure. However, since $\phi$ is a Jordan isomorphism, we have $x y+y x=2$ and $x y x=x$. Thus $(x y)^{2}=x y$ and $(y x)^{2}=y x$, and we have in turn $4-4 x y+x y=(2-x y)^{2}=2-x y, x y=1, y x=1$. Since $A^{+}$is of type- 0 , it now follows that $W$ is of type-0. Since $a b a \in A^{+}\left(a, b \in A^{+}\right)$and $\phi$ is a Jordan isomorphism, we have $x y x \in W(x, y \in W)$. By Theorem $4, W$ is locally multiplicative, and Theorem 3 of (2) now shows that $R$ satisfies (ii) and (iii). That $R$ satisfies (i) and (iv) is clear from the boundedness of $\phi$ and $\phi^{-1}$ and from the inequality $\left\|\left(1+a^{2}\right)^{-1}\right\| \leqslant 1(a \in A)$.

The next lemma may appear obvious at first sight but involves the difficulty that the spectra of elements are defined in terms of the complex Banach algebras $B$ and $B L(H)$ whereas the Jordan isomorphism is defined only on the Jordan subalgebra $R$.

Lemma 6. Let $R$ be a Jordan subalgebra of $B$ containing 1 , and let $\phi$ be $a$ bicontinuous isomorphism of $R$ onto a JC-algebra. Then

$$
\operatorname{Sp}(\phi(x))=\operatorname{Sp}(x) \quad(x \in R) .
$$

Proof. Let $W=\left\{x \in R: S p(x) \subset R^{+}\right\}$. By Corollary 5 and (2, Theorem 3), $W$ is a closed type-0 locally multiplicative wedge. Also $\left\{\left\|(1+w)^{-1}\right\|: w \in W\right\}$ is bounded. Therefore, by Theorem 1 , the spectral radius is a norm on $R$ equivalent to the given norm, and so there exists a constant $\kappa>0$ such that

$$
r(x) \geqslant \kappa\|x\| \quad(x \in R) .
$$

We deduce that

$$
x, y \in R, x y=y x \Rightarrow\|x+i y\| \geqslant \frac{\kappa^{2}}{4}(\|x\|+\|y\|) .
$$

Given $x, y \in R$ with $x y=y x$, we have

$$
(\|x\|+\|y\|)\|x+i y\| \geqslant\|x-i y\|\|x+i y\| \geqslant\left\|x^{2}+y^{2}\right\| \geqslant r\left(x^{2}+y^{2}\right) \geqslant r\left(x^{2}\right) \geqslant \kappa^{2}\|x\|^{2} .
$$

Similarly $(\|x\|+\|y\|)\|x+i y\| \geqslant \kappa^{2}\|y\|^{2}$, and so

$$
(\|x\|+\|y\|)\|x+i y\| \geqslant \frac{\kappa^{2}}{2}\left(\|x\|^{2}+\|y\|^{2}\right) \geqslant \frac{\kappa^{2}}{4}(\|x\|+\|y\|)^{2},
$$

which proves (8).

We prove next that

$$
x \in R \cap \operatorname{Inv}(B) \Rightarrow x^{-1} \in R .
$$

Let $x \in R \cap \operatorname{Inv}(B)$, and let $C$ be the least closed complex subalgebra of $B$ containing 1 and $x$. Since $\operatorname{Sp}(x) \subset R$, the spectrum of $x$ relative to $C$ coincides with its spectrum relative to $B$. Therefore $x^{-1} \in C$, and there exist real polynomials $p_{n}, q_{n}$ in $x$ such that $x^{-1}=\lim _{n \rightarrow \infty}\left(p_{n}+i q_{n}\right)$. It follows from (8) that there exist $p, q \in R \cap C$ such that $\lim _{n \rightarrow \infty} p_{n}=p, \lim _{n \rightarrow \infty} q_{n}=q$. Thus $x^{-1}=p+i q$. We have $p x, q x \in R \cap C$ and $(p x-1)+$ $i q x=0$. Therefore, by (8), $p x-1=0, x^{-1}=p \in R$; (9) is proved.

Given $x, y \in R$ with $x y=y x=1$, we have, as in the proof of Corollary 5, 
$\phi(x) \phi(y)=\phi(y) \phi(x)=1$. It follows, by (9) that if $x \in R \cap \operatorname{Inv}(B)$, then $\phi(x)$ is invertible in $B L(H)$. Finally, since $\operatorname{Sp}(x) \subset \boldsymbol{R}$ for all $x \in R$, we now have $\operatorname{Sp}(\phi(x))=$ $\operatorname{Sp}(x)$.

We now consider the characterisation of bicontinuous isomorphic images of $J C$-algebras in terms of a submultiplicative property of the spectral radius in place of the subadditive property in Corollary 5.

Theorem 7. Let $R$ be a Jordan subalgebra of $B$ containing 1 . Then $R$ is bicontinuously isomorphic to a JC-algebra if and only if it satisfies

(i) $R$ is closed,

(ii) $\mathrm{Sp}(x) \subset \boldsymbol{R} \quad(x \in R)$,

(iii) $x, y \in R, \lambda \in R \cap \operatorname{Sp}(x y) \Rightarrow|\lambda| \leqslant r(x) r(y)$,

(iv) $\left\{\left\|\left(1+x^{2}\right)^{-1}\right\|: x \in R\right\}$ is bounded.

Proof. Suppose first that conditions (i)-(iv) hold, and let $W=\left\{x \in R: \operatorname{Sp}(x) \subset R^{+}\right\}$. Minor modifications of the proof of (2, Theorem 8) show that $W$ is a closed type-0 locally multiplicative wedge and that $R=W-W$. Thus Corollary 2 gives the required bicontinuous isomorphism.

Suppose conversely that $\phi$ is a bicontinuous isomorphism of $R$ onto a $J C$-algebra $A$. We prove first that

$$
x, y, z \in R \Rightarrow \mathrm{Sp}(z(x y-y x) z) \subset i R .
$$

Given $x, y, z \in R$, let $a=\phi(x), b=\phi(y), c=\phi(z)$. We have

$$
(z(x y+y x) z)^{2}-(z(x y-y x) z)^{2}=2 z x y z^{2} y x z+2 z y x z^{2} x y z
$$

and, in turn, $y z^{2} y \in R, x y z^{2} y x \in R, z x y z^{2} y x z \in R$. Therefore $(z(x y-y x) z)^{2} \in R$, and, since $\phi$ is a Jordan isomorphism,

$$
\begin{aligned}
\phi\left((z(x y-y x) z)^{2}\right) & =\phi\left((z(x y+y x) z)^{2}\right)-2 \phi\left(z x y z^{2} y x z\right)-2 \phi\left(z y x z^{2} x y z\right) \\
& =(c(a b+b a) c)^{2}-2 c a b c^{2} b a c-2 c b a c^{2} a b c \\
& =(c(a b-b a) c)^{2} .
\end{aligned}
$$

Since ic $(a b-b a) c$ is self-adjoint, we have $\operatorname{Sp}\left((c(a b-b a) c)^{2}\right) \subset-\boldsymbol{R}^{+}$. Therefore, by Lemma $6, \operatorname{Sp}\left((z(x y-y x) z)^{2}\right) \subset-R^{+}$, and (10) is proved.

Let $W=\phi^{-1}\left(A^{+}\right)$. Then $W$ is a closed type-0 locally multiplicative cone and $W=\left\{x \in R: \operatorname{Sp}(x) \subset \boldsymbol{R}^{+}\right\}$. Let $x, y \in R$ and $\lambda \in R$ with $\lambda>r\left(\frac{1}{2}(x y+y x)\right)$. Then $2 \lambda-$ $(x y+y x) \in W \cap \operatorname{Inv}(B)$ and so $2 \lambda-(x y+y x)=z^{-2}$ with $z \in W$. Therefore

$$
\begin{aligned}
2 \lambda-2 x y & =2 \lambda-(x y+y x)-(x y-y x) \\
& =z^{-1}\{1-z(x y-y x) z\} z^{-1}
\end{aligned}
$$

By (10), it follows that $\lambda-x y \in \operatorname{Inv}(B)$; and we have proved that $\lambda \notin \operatorname{Sp}(x y)$ whenever $\lambda>r\left(\frac{1}{2}(x y+y x)\right)$. Replacing $x$ by $-x$, we see that $\lambda \notin S p(x y)$ whenever $-\lambda>$ $r\left(\frac{1}{2}(x y+y x)\right)$, and so 


$$
\lambda \in R \cap S p(x y) \Rightarrow|\lambda| \leqslant r\left(\frac{1}{2}(x y+y x)\right) .
$$

But, by (2, Theorem 4), $r\left(\frac{1}{2}(x y+y x)\right) \leqslant r(x) r(y)$, and so (iii) is proved; and (i), (ii), (iv) have been proved in Corollary 5.

I owe the following example to $\mathrm{M}$. A. Youngson. This not only shows that we can have $W \cap(-W)=\{0\}$ without having boundedness of $\left\{\left\|(1+w)^{-1}\right\|: w \in W\right\}$, but also settles a question asked in $(2, p .247)$ concerning the existence of square roots.

Example 8. Take $B$ to be the complex Banach algebra $C_{1}[0,1]$ of all continuous complex functions on $[0,1]$ with continuous first derivatives there, with the usual norm $\|x\|=\sup \{|x(s)|: 0 \leqslant s \leqslant 1\}+\sup \left\{\left|x^{\prime}(s)\right|: 0 \leqslant s \leqslant 1\right\}$. Let $V$ be the set of all nonnegative real valued functions belonging to $B$. Plainly $V$ is a closed type-0 locally multiplicative cone. As is no doubt well known, $V$ is not a normal cone; for example consider $u, v \in V$ given by $u(s)=s^{n}, v(s)=1-s^{n}(0 \leqslant s \leqslant 1)$. It follows that the set $\left\{\left\|(1+w)^{-1}\right\|: w \in V\right\}$ is not bounded, as can also be verified directly without difficulty. Moreover, the element $w$ of $V$ given by $w(s)=s(0 \leqslant s \leqslant 1)$ has no square root in $V$; so that $W \cap(-W)=\{0\}$ is not sufficient to give the existence of square roots of elements of $W$ (see (2, p. 247)). In this connection it should also be noted that if elements of $W$ have at most one square root in $W$, then $W \cap(-W)=\{0\}$. For if $h \in W \cap(-W)$, then $h^{2}$ is an element of $W$ with the square roots $h$ and $-h$ in $W$.

In the light of Example 8 it is of interest, when $W \cap(-W)=\{0\}$, to consider the completion of $W-W$ with respect to the spectral radius norm. By using the full force of the characterisation of special $J B$-algebras in (1), we show that the completion is still a special $J B$-algebra.

Theorem 9. Let $W$ be a closed type-0 locally multiplicative cone in $B$, and let $R=W-W$. Then $r$ is a norm on $R$, and the completion of $R$ with the natural extension of $r$ and of the Jordan product on $R$, is a special JB-algebra.

Proof. Since $W \cap(-W)=\{0\}$, Theorems 3 and 4 in (2) show that $r$ is a norm on the Jordan algebra $R$ and that

$$
r(x \circ y) \leqslant r(x) r(y) \quad(x, y \in R),
$$

where $x \circ y=\frac{1}{2}(x y+y x)$. Let $S$ denote the completion of $R$ with respect to the norm $r$ and let $r$ denote also the natural extension of the norm $r$ to $S$. Then $S$ with the extended norm $r$ is a real Banach space. By (11), the Jordan product extends by continuity to a mapping: $S \times S \rightarrow S$ which we denote also by $x \circ y$. It is routine to check that $x \circ y$ is a distributive and commutative product on $S$, that $\alpha(x \circ y)=(\alpha x) \circ y=$ $x \circ(\alpha y)$ for $\alpha \in R$ and that $x^{2} \circ(y \circ x)=\left(x^{2} \circ y\right) \circ x$. Thus $S$ with this product is a Jordan algebra.

Given $x, y \in S$, choose Cauchy sequences $\left\{x_{n}\right\},\left\{y_{n}\right\}$ in $R$ corresponding to $x, y$ respectively. Then, since the proof of (4) does not use the boundedness of the set $\left\{(1+w)^{-1}: w \in W\right\}$, we have

$$
r\left(x^{2}+y^{2}\right)=\lim _{n \rightarrow \infty} r\left(x_{n}^{2}+y_{n}^{2}\right) \geqslant \lim _{n \rightarrow \infty} r\left(x_{n}^{2}\right)=r\left(x^{2}\right),
$$


and $r\left(x^{2}\right)=\lim _{n \rightarrow \infty} r\left(x_{n}^{2}\right)=\lim _{n \rightarrow \infty}\left(r\left(x_{n}\right)\right)^{2}=(r(x))^{2}$. We have now proved that $(S, r)$ is a $J B$-algebra. To prove that $S$ is special, let $f$ be a real polynomial in three variables that vanishes on all special Jordan algebras but not on $M_{3}^{8}$, the exceptional formally real simple Jordan algebra of finite dimension. Then $f(x, y, z)=0$ for all $x, y, z \in R$ and therefore also (by continuity) for all $x, y, z \in S$. Therefore, by (1, Theorem 9.4), $S$ is a special $J B$-algebra.

Corollary 10. Let $W, R$ be as in Theorem 9. Then the Jordan algebra $R$ is isomorphic to a dense Jordan subalgebra of a JC-algebra.

Proof. Let $S$ be the completion of $R$ with respect to the norm $r$. Then $S$ with the extended norm $r$ is a special $J B$-algebra and is therefore isometrically isomorphic to a $J C$-algebra.

Acknowledgement. I am indebted to E. M. Alfsen, F. W. Shultz, and E. Størmer for a preprint copy of (1), which is the essential basis for this note.

\section{REFERENCES}

(1) E. M. Alfsen, F. W. Shultz, and E. Størmer, A Gelfand-Neumark theorem for Jordan algebras, Advances in Math. 28 (1978), 11-56.

(2) F. F. BONSALL, Locally multiplicative wedges in Banach algebras, Proc. London Math. Soc. (3) 30 (1975), 239-256.

(3) E. STøRMER, On the Jordan structure of $C^{*}$-algebras, Trans. Amer. Math. Soc. 120 (1965), 438-447.

UNIVERSITY OF EDINBURGH 